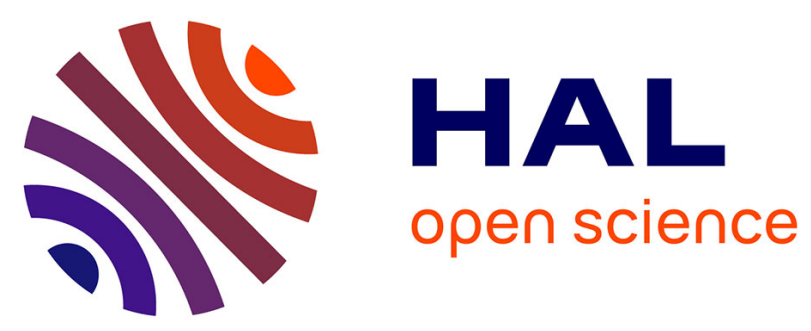

\title{
Propriétés magnétiques des alliages dilués. Interactions magnétiques et antiferromagnétisme dans les alliages du type métal noble-métal de transition
}

\author{
Annie Blandin, J. Friedel
}

\section{- To cite this version:}

Annie Blandin, J. Friedel. Propriétés magnétiques des alliages dilués. Interactions magnétiques et antiferromagnétisme dans les alliages du type métal noble-métal de transition. J. Phys. Radium, 1959, 20 (2-3), pp.160-168. 10.1051/jphysrad:01959002002-3016000 . jpa-00236009

\section{HAL Id: jpa-00236009 https://hal.science/jpa-00236009}

Submitted on 1 Jan 1959

HAL is a multi-disciplinary open access archive for the deposit and dissemination of scientific research documents, whether they are published or not. The documents may come from teaching and research institutions in France or abroad, or from public or private research centers.
L'archive ouverte pluridisciplinaire HAL, est destinée au dépôt et à la diffusion de documents scientifiques de niveau recherche, publiés ou non, émanant des établissements d'enseignement et de recherche français ou étrangers, des laboratoires publics ou privés. 


\title{
PROPRIÉTÉS MAGNÉTIQUES DES ALLIAGES DILUÉS. INTERACTIONS MAGNÉTIQUES ET ANTIFERROMAGNÉTISME DANS LES ALLIAGES DU TYPE MÉTAL NOBLE-MÉTAL DE TRANSITION
}

\author{
Par A. BLANDIN et J. FRIEDEL, \\ Centre de Physique des Solides, Paris $\left({ }^{*}\right)$.
}

\begin{abstract}
Résumé. - On étudie la structure électronique des impuretés de transitıon dans les alliages désordonnés à faible concentration. On en déduit une classification des propriétés magnétiques de ces alliages. Un modèle d'interaction magnétique entre impuretés est proposé, pour rendre compte des propriétés antiferromagnétiques de certains alliages (Cu Mn).
\end{abstract}

Abstract. - The electronic structure of disordered alloys of a transition elements at low concentrations is investigated and a classification of their magnetic properties is obtained. A model of magnetic interaction between impurities is proposed to explain the antiferromagnetic properties of some such alloys ( $\mathrm{Cu} \mathrm{Mn).}$

Des résultats expérimentaux récents sur les propriétés magnétiques [1], [2], [3] et électriques [3], [4], [5] des alliages désordonnés de métaux nobles $(\mathrm{Cu}, \mathrm{Ag}, \mathrm{Au})$ avec les métaux de transition $(\mathrm{Mn}, \mathrm{Cr})$ à faible concentration de métal de transition, ont mis en évidence l'existence d'interaction à grande distance entre atomes magnétiques.

Le but de cette communication est d'étudier la structure électronique des impuretés magnétiques dans les métaux, d'en déduire un modèle d'interaction et d'exposer les essais tentés pour interpréter, à partir de ce modèle, les résultats expérimentaux.

1. Structure électronique des impuretés dissoutes dans un métal. - 1.1. Notion D'ÉTAT LIÉ virTUEL. - La notion d' " états liés virtuels",

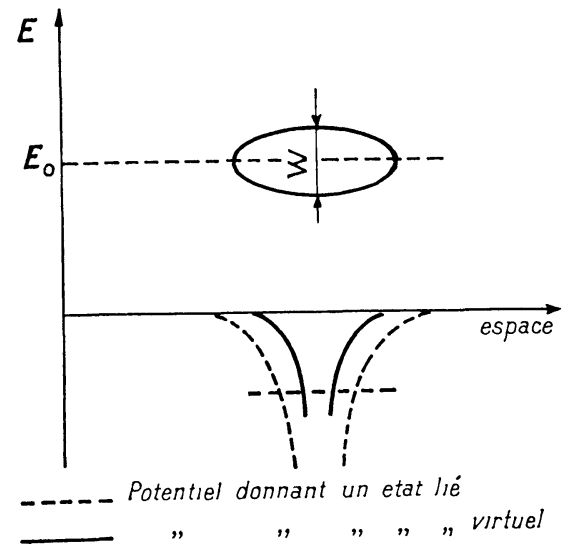

FIG. 1.

connue dans la diffusion des électrons par les atomes libres, a été étendue aux cas des impuretés

(*) 60, boulevard Saint-Michel, Paris $\left(6^{\mathrm{e}}\right)$. de transition dissoutes dans les métaux [6], [7]. On assimile les électrons du réseau non perturbé à un gaz d'électrons libres de niveaux de Fermi $E_{\mathrm{F}}$. L'élément de transition, à $2 Z$ électrons $d$, ne possède pas un potentiel suffisamment attractif pour avoir des niveaux liés $d\left({ }^{1}\right)$. L' " état lié virtuel " a une énergie tombant dans le continuum des états étendus (fig. 1). Par suite de ses résonances avec les états étendus, ce n'est plus un vrai état lié, mais seulement un paquet d'ondes centré sur l'impureté de transition, étentu en énergie au voisinage de l'énergie moyenne $E_{0}$, et dans l'espace autour de l'atome d'impureté. Les amplitudes des composantes $d$ des états étendus d'énergie voisine de $E_{0}$ sont grandes sur l'atome d'impureté et donnent lieu à un excès de charge à peu près égal à celui qu'aurait l'état lié s'il existait.

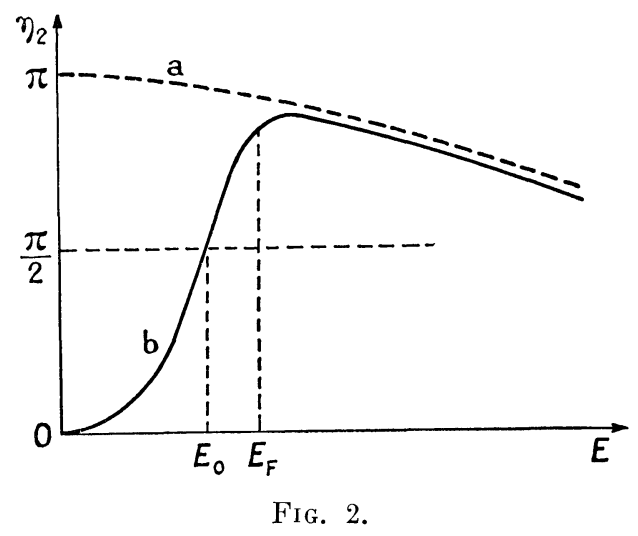

Les déphasages $\eta_{l}(E)$ des composantes $d$ des états étendus sont donnés schématiquement par la courbe $a$ (fig. 2) pour un état lié et pour la courbe $b$ (fig. 2) pour un état lié virtuel. Le zéro d'énergie

(1) Dans tout ce qui suit, on supposera le couplage spinorbite négligeable. 
est pris ici arbitrairement au bas de la bande de conductibilité du métal. Il est montré en appendice, en choisissant une forme de potentiel simple, que les déphasages varient bien comme l'indique la figure 2. Dans la suite de l'exposé, on supposera donnée la courbe de déphasage en fonction de l'énergie.

Soit $\alpha$ le nombre d'électrons de conduction par atome dans la matrice. En supposant que l'atome de transition a $2 Z+1$ électrons (par exemple $2 Z$ électrons $d$ et un électron $s$ ), la chargeionique à écranter est alors $(2 Z+1-\alpha)\left({ }^{2}\right)$.

D'après la règle de somme de Friedel, on a alors :

$$
(2 Z+1-\alpha)=\frac{2}{\pi} \sum_{l}(2 l+1) \eta_{l}\left(E_{F}\right)
$$

où $\eta_{l}\left(E_{\mathrm{T}}\right)$ sont les déphasages des composantes sphériques' $l$ au niveau de Fermi $E_{\mathrm{F}}$.

Dans le cas des métaux nobles, $\alpha=1$, et on peut supposer les déphasages $\eta_{l}$ petits pour $l \neq 2$, c'est-à-dire que tout l'écran est produit par le niveau virtuel $d$. La condition de Friedel devient : $Z_{2}\left(E_{\mathrm{F}}\right) \simeq Z \quad$ si $\quad Z_{l}(E)=\frac{2 l+1}{\pi} \eta_{l}(E) \quad$ est la charge écrantée par les électrons de symétrie $l$ et d'énergie inférieure à $E$.

Dans le cas de l'aluminium, $\alpha=3$ et il est raisonnable de penser que les composantes sphériques autres que $d$, produisent le même écran pour toutes les impuretés de transition et aussi pour les métaux nobles.

En supposant $\eta_{l}=0$ pour $l>2$, on a ainsi

$$
\begin{aligned}
& \frac{2}{\pi}\left[\eta_{0}\left(E_{\mathbf{F}}\right)+3 \eta_{1}\left(E_{\mathbf{F}}\right)\right] \simeq-2 \\
& \frac{5}{\pi} \eta_{2}\left(E_{\mathbf{F}}\right)=Z_{2}\left(E_{\mathbf{F}}\right) \simeq Z .
\end{aligned}
$$

1.2. DÉCOUPLAGE DES DEUX DIREGTIONS DE SPIN.

a) Cas d'un atome. - Dans un atome possédant $2 Z$ électrons $d$ formant une couche incomplète, on sait que l'on gagne de l'énergie en découplant les deux directions de spin. Si $p$ est le nombre d'électrons (ou de trous) non appariés, d'une direction de spin, l'énergie d'échange stabilisant cet état est : $\Delta E_{\text {ech. }}=-\frac{1}{2} p(p-1) \Delta E$ où $\Delta E$ est le gain moyen d'énergie quand, pour deux électrons, on passe de la position antiparallèle de spin à la position parallèle. $\Delta E$ peut être considéré comme presque constant pour l'ensemble des éléments de transition du scandium au nickel et égal à environ $0,6 \mathrm{eV}$.

Lors du découplage des spins, la variation totale d'énergie se réduit, au premier ordre, à la variation d'énergie d'échange $\Delta E$ éch. donnée plus haut.

(2) On se place alors dans le système d'unités atomiques $\hbar=m=e=1$.
Comme cette énergie est négative, on obtiendra toujours, pour un atome libre, le découplage maximum entre les deux directions de spin. C'est la règle de Hund.

b) Cas d'un niveau lié virtuel. - La charge d'écran $Z_{2}(E)$ (fig. $3 a$ ) peut se découpler en $Z_{2} \downarrow(E)$ et $Z_{2} \uparrow(E)$ pour chaque direction de spin (fig. $3 b$ et $c$ ). L'état virtuel d'énergie moyenne $E_{0}$ donne deux états liés virtuels d'énergies moyennes $E_{0} \uparrow$ et $E_{0} \downarrow$. Soit

$$
p=Z_{2} \uparrow\left(E_{\mathbf{F}}\right)-Z_{2} \downarrow\left(E_{\mathbf{F}}\right)
$$

le découplage des spins. La variation d'énergie d'échange due à ce découplage est

$$
\Delta E_{\mathrm{ech}}=-\frac{1}{2} p^{2} \Delta E
$$

Pour obtenir cette expression, on assimile les

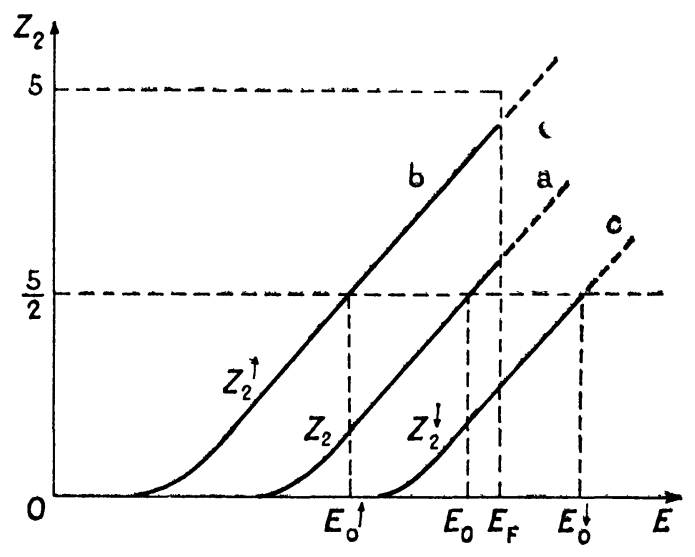

FIG. 3.

états liés virtuels à de vrais états liés dont toute la charge serait concentrée à l'énergie moyenne $E_{0}$ sans découplage, et aux énergies moyennes $E_{0} \uparrow$ et $E_{0} \downarrow$ avec découplage ( $f i g .3$ ). Du fart que les états virtuels ont une certaine largeur en énergie, la variation totale d'énergie $\Delta \varepsilon$ comprend donc, en plus du terme d'échange $\Delta E_{\text {ech}}$, un terme d'énergie individuelle : $\Delta E_{i}$

$$
\Delta \varepsilon=\Delta E_{\text {ech }}+\Delta E_{i}
$$

$\Delta E_{\imath}$ est la variation lors du découplage de la somme des énergies individuelles par rapport aux énergies moyennes $E_{0}, E_{0} \uparrow$ et $E_{0} \downarrow$. Elle se calcule simplement en considérant que la charge d'écran d'un état lié virtuel forme une "bande " dont la densité des états par unité d'énergie est $\mathrm{d} Z_{2}(E) / \mathrm{d} E$. Cette bande est représentée figure 4. Sans découplage, elle est remplie jusqu'au niveau $E_{F}$; avec découplage, elle est remplie jusqu'à des niveaux $E_{\mathrm{F}} \uparrow$ et $E_{\mathrm{F}} \downarrow$ tels que la condition d'écran discutée au § 1.1 soit conservée. D'où finalement :

$$
Z_{2}\left(E_{\mathrm{F}} \uparrow\right)-Z_{2}\left(E_{\mathrm{F}}\right)=Z_{2}\left(E_{\mathrm{F}}\right)-Z_{2}\left(E_{\mathrm{F}} \downarrow\right)=\frac{1}{2} p .
$$


La condition de découplage $\Delta \varepsilon<0$ donne alors, pour un découplage $p$ in finiment petit $\left({ }^{3}\right)$

$$
2 \Delta E \frac{\mathrm{d} Z_{2}\left(E_{\mathbf{F}}\right)}{\mathrm{d} E}>1 \text {. }
$$

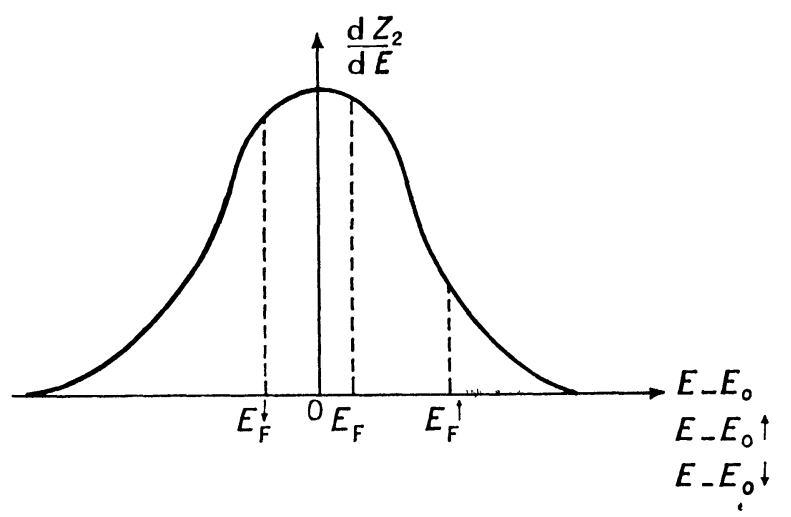

FIG. 4.

c) Étude de découplage sur un modèle simple d'état virtuel lié. - En supposant que la méthode employée ci-dessus est encore valable pour un découplage fort, et en se donnant une forme d'état lié virtuel simple mais physiquement acceptable, on peut alors chercher quel est le découplage le plus stable pour une charge donnée d'écran par électron $d, 2 Z_{2}$.

Les calculs ont été faits en prenant un niveau lié virtuel de forme parabolique :

et

$$
\frac{\mathrm{d} Z_{2}}{\mathrm{~d} E}=\frac{15}{4 W}\left(1-\frac{E^{2}}{W^{2}}\right) \text { pour }|E| \leqslant W
$$

$$
\frac{\mathrm{d} Z_{2}}{\mathrm{~d} E}=0 \text { pour }|E| \geqslant W
$$

l'origine des énergies a été prise ici au milieu de la bande, et $\mathrm{d} Z_{2} / \mathrm{d} E$ normée de telle sorte que

$$
\int_{-W}^{+W} \frac{\mathrm{d} Z_{2}}{\mathrm{~d} E} \mathrm{~d} E=5
$$

On écrit que le nombre des électrons d'écran est égal à $2 Z_{2}$; d'autre part, la condition pour avoir le découplage maximum peut s'écrire :

$\left(E_{\mathrm{F}} \uparrow-E_{\mathrm{F}} \downarrow\right)=2 p \Delta E$ avec $E_{\mathbf{F}} \uparrow \leqslant W$ et $E_{\mathbf{F}} \downarrow \geqslant-W$.

Après des calculs sans difficultés qui ne seront pas reproduits ici, on obtient les résultats suivants ( $f$ g. 5): en fonction de $W / \Delta E$ on porte $2 Z_{2}$ (nombre d'électrons ou de trous dans le niveau $d$ ).

(3) On peut remarquer que cette discussion du découplage est identıque à celle faite par Stoner pour le ferromagnétısme des métaux purs. Dans les deux cas, il n'est pas possible d'obtenir l'énergie de découplage en comparant simplement la somme des énergies individuelles des électrons. Cette procédure, appliquée à la figure 3, conduirart a un résultat erroné parce qu'elle compterait deux fois les termes de correlation entre les electrons.
On distingue alors 3 régions : en $\mathrm{A}$, découplage total, en $\mathrm{B}$, découplage partiel, en $\mathrm{C}$, décoùplage nul.

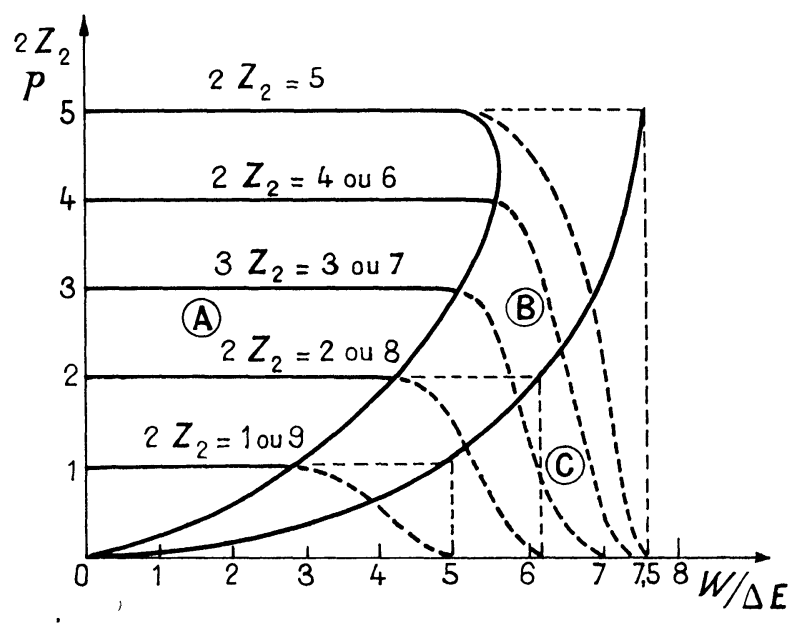

FIG. 5 .

On en déduit les courbes donnant le découplage $p$ en fonction de $W \mid \Delta E$ pour chaque valeur de $2 Z_{2}$. On voit sur la figure 5 que la condition de découplage $W \mid \Delta E r<2 Z_{2}$ donnée précédemment [7] est un peu trop restrictive.

\subsection{Applichtion aux Propriétés des alliages} A FAIBLE CONGENTRATION DE MÉTAL DE TRANSITION. - D'après la discussion qui précède, les impuretés de transition ne doivent pas toujours suivre la règle de Hund : il n'y a découplage des deux directions de spins que si le niveau $d$ a une largeur $W$ assez faible, ou s'il contient un nombre d'électrons ou de trous positifs assez voisin de 5 .

On sait, d'autre part, que la largeur $W$ d'un état lié virtuel $d$ croît avec son énergie moyenne $E_{0}$ (pour des énergies pas trop fortes) [7]. Ceci est vérifié sur le cas particulier traité en appendice. Les impuretés de transition, dont les couches $d$ sont incomplètes, ont nécessairement des énergies moyennes $E_{0}$ de l'ordre du niveau de Fermi de la matrice : leur largeur $W$ doit croître avec l'énergie de Fermi de la matrice, donc avec sa valence. On s'attend ainsi à ce que le découplage des spins soit d'autant moins facile que la matrice a une valence plus élevée : il ne doit également se produire que pour des impuretés ayant un nombre suffisant d'électrons ou de trous $d$. C'est ce que semble confirmer l'étude des propriétés physiques de ces alliages.

a) Résistivité des alliages à base d'Al-Au-Cu. Les augmentations de résistivité par rapport aux métaux purs des alliages d'éléments de transition dans l'aluminium, le cuivre et l'or, ont été expli- 
quées sur la base du modèle précédent [6], [7]. L'augmentation $\lambda$ par \% d'impuretés est [8]

$$
\begin{gathered}
\lambda=\frac{4 \pi}{\alpha k_{F}} \sum l \sin ^{2}\left(\eta_{l-1}-\eta_{l}\right) \\
\text { s'il n'y a pas découplage } \\
\lambda=\frac{2 \pi}{\alpha k_{F}} \sum_{l} l\left[\sin ^{2}\left(\eta_{l}^{\uparrow}-1-\eta_{l}^{\uparrow}\right)+\sin ^{2}\left(\eta_{l}-_{-1}-\eta_{l}^{\downarrow}\right)\right] \\
\text { s'il y a découplage }\left(^{4}\right)
\end{gathered}
$$

où $k_{\mathrm{F}}$ est le vecteur d'onde au niveau de Fermi, $\alpha$ le nombre d'électrons de conduction par atome dans la matrice. On peut en déduire les résultats suivants : pour les alliages à base d'aluminium, le découplage est nul ou très faible même pour les éléments centraux de la série $(\mathrm{Cr}, \mathrm{Mn}, \mathrm{Fe})$, la charge d'écran produite par les électrons $d$ étant voisine de $2 Z$. Pour les alliages à base de cuivre et or, il y a un fort découplage pour les éléments centraux $(\mathrm{Mn}, \mathrm{Cr})$. Pour les éléments où $2 Z$ est petit $(\mathrm{Sc})$ ou voisin de $10(\mathrm{Co}, \mathrm{Ni})$, on ne peut pas conclure.

b) Propriétés magnétiques des alliages solides à base d'Al-Au-Cu. - Sans découplage des deux directions de spin, on doit observer un paramagnétisme de Pauli pratiquement constant avec la température ; avec découplage des directions de spins, un paramagnétisme de Langevin de haute température $\chi \simeq C / T$ avec une constante $C$ correspondant à un nombre de magnétons de Bohr compris entre 0 et 5,9 (pour un spin $S=5 / 2$ ).

Les composés définis $\mathrm{Ni} \mathrm{Al}_{3}, \mathrm{Co}_{2} \mathrm{Al}_{5}, \mathrm{Co}_{4} \mathrm{Al}_{13}$, $\mathrm{Mn} \mathrm{Al}_{6}, \quad \mathrm{Cr} \mathrm{Al}_{7}$ présentent un paramagnétisme très peu variable avec la température [9], [10]. Les alliages dilués $\mathrm{Al} \mathrm{Mn}$ [11] ont également une susceptibilité paramagnétique constante. On peut donc en déduire que dans l'aluminium et à une dilution suffisante pour que les atomes de transition n'interagissent que faiblement, les couches $d$ de l'élément de transition ont leurs deux directions de spin également remplies (pas de découplage).

La susceptibilité magnétique des alliages à base de métal noble, contenant du chrome, du manganèse et du fer [13], [14], [15], met en évidence un moment magnétique permanent correspondant à un découplage presque total. Au contraire, les alliages $\mathrm{Cu} \mathrm{Ni}$ [17], [18] ont un paramagnétisme constant avec un petit terme en $1 / T$ dont le coefficient varie comme le cube de la concentration. Ce terme peut être interprété en supposant que seuls les groupements de trois atomes de nickel voisins sont susceptibles de provoquer un découplage de spins.

Enfin un alliage $M g$ Mn a été étudié [2]: il possède une susceptibilité inversement proportionnelle à la température correspondant au

(4) On n'a tenu compte ici que des diffusions élastiques. Si l'on fait intervenir les diffusions où l'électron change de direction de spin, il faut introduire un facteur correctif qui n'a que peu d'importance ici [12]. moment magnétique d'un spin $S=3 / 2$, c'est-àdire un découplage partiel. L'ensemble de ces résultats concorde avec ceux de $1.3 a$, et précise dans le cas des métaux nobles que l'on a pour le nickel (et peut-être le cobalt) un découplage nul.

c) Propriétés magnétiques des alliages liquides. Les mesures de susceptibilité magnétique des alliages liquides ont été interprétées [19] par des formules de Curie $\chi=C /(T-\theta)(\chi$ susceptibilité par gramme de métal de transition). On rencontre deux cas extrêmes : ou bien $\mathrm{C}$ est à peu près constant en fonction de la concentration et correspond à un spin atomique raisonnable et $\theta$ est petit (c'est le cas de Au Mn par exemple); ou bien C augmente rapidement quand la concentration diminue et $\theta$ prend des valeurs négatives très élevées (plusieurs milliers de degrés $K$; c'est le cas de $\mathbf{A u ~ N i ) . ~ L e s ~ m e s u r e s ~ a y a n t ~ e ́ t e ́ ~ e f f e c t u e ́ e s ~ s u r ~ u n ~}$ intervalle de température restreint ( 300 à 400 oK), on ne peut déduire de résultats précis, en particuller pour les valeurs de $\theta$; mais de très fortes valeurs de $\theta$ à faible concentration sont incompréhensibles. Les résultats s'interprètent beaucoup plus facilement dans le modèle proposé ici, en comparant les résultats à une formule du type $\chi=a+b / T$. Dans le premier cas, $a \simeq 0$; dans le deuxième, on trouve un paramagnétisme constant : $b \simeq 0$.

Dans les alliages à base d'or, on trouve un paramagnétisme en $1 / T$ pour le manganèse et le fer, un paramagnétisme constant pour le nickel. Le cas du cobalt est intermédiaire. La configuration électronique des impuretés dans l'alliage liquide est voisine de la configuration à l'état solide (le niveau de Fermi des électrons de conduction reste sensiblement le même) ; on le vérifie sur les propriétés magnétiques à l'état liquide et à l'état solide.

\begin{tabular}{c|c|c|c|c|c|} 
Solvant & $\mathrm{Au}$ & $\mathrm{Cu}$ & $\mathrm{Mg}$ & $\mathrm{Zn}$ & $\mathrm{Al}$ \\
\hline$E_{\mathrm{F} \text { e eV }}$ & 5,5 & 7 & 7,5 & 9,5 & 13 \\
\hline$\frac{W}{\Delta E} \sim$ & 3 & $<5$ & 5 & 6 & $>7$ \\
\hline $\mathrm{Cr}_{\mathrm{N}}$ & + & + & & & - \\
$\mathrm{Mn}$ & + & + & + & + & - \\
$\mathrm{Fe}$ & + & + & & - & - \\
$\mathrm{Co}$ & + & & & & - \\
$\mathrm{Ni}$ & - & - & & - & - \\
\hline
\end{tabular}

Fig. 6.

Le zinc, solvant intermédiaire entre le cuivre et l'aluminium, donne des alliages liquides à paramagnétisme en $1 / T$ pour le manganèse, très peu 
variable avec le fer et constant avec le nickel. Dans ce cas, le découplage des spins n'interviendrait que pour le manganèse.

1.4. Conclusion. - On peut alors dresser un tableau (fig. 6) en indiquant par + ou - s'il y a ou non découplage.

D'après les calculs du $§ 1.2 c$, les résultats expérimentaux demandent que $W \mid \Delta E$ soit supérieur à 7 dans les alliages d'aluminium et de l'ordre de 3 à 5 dans le cuivre et l'or. De telles valeurs croissent bien avec l'énergie de Fermi de la matrice (de $13 \mathrm{eV}$ dans l'aluminium à $5,5 \mathrm{eV}$ dans l'or). Elles sont raisonnables d'un point de vue théorique [7].

Les largeurs calculées en appendice sont proportionnelles à l'énergie moyenne $E_{0}$ du niveau lié virtuel ; elles valent 3 et $7 \mathrm{eV}$ pour des énergies moyennes de $5,5 \mathrm{eV}$ et $13 \mathrm{eV}$, ce qui est en accord satisfaisant avec les valeurs de $W \mid \Delta E$ de la figure 6 .

Le modèle proposé ici rend bien compte qualitativement d'un ensemble de faits expérimentaux qui peuvent être ainsi reliés de façon cohérente. Pour interpréter quantitativement ces résultats, il faudrait une meilleure connaissance de la forme des états liés virtuels et de leur découplage $\left(^{5}\right)$.

D'autre part, jusqu'à présent, les interactions entre impuretés ont été négligées. Le but de la deuxième partie est de montrer que le modèle est susceptible de rendre compte d'interaction, même à grande distance, entre atomes magnétiques.

2. Interactions magnétiques entre impuretés. On observe expérimentalement, même à basse concentration, des particularités dans les propriétés magnétiques et électriques des alliages des métaux nobles avec les métaux de transition. En particulier l'intérêt sera centré sur les alliages Cu Mn qui ont donné lieu à de nombreuses études récentes [1], [2], [3], [4], [5].

2.1. Résultats eXPÉrimentaux POUR LES alliages $\mathrm{Cu} \mathrm{Mn}$. - La susceptibilité [1], [3] à

(5) Une méthode de détermination de la forme des états liés virtuels est la mesure des chaleurs spécifiques électroniques des alliages. La chaleur spécifique électronique est proportionnelle à la densité des états du niveau de Fermi $n\left(E_{\mathbf{F}}\right)$. La matrice ayant la densité d'états $n_{0}(E)$, si $x$ est la concentration en impuretés et $Z(E)$ la charge d'écran des électrons d'énergie inférieure à $E$, on a :

$$
n\left(E_{\mathbf{F}}\right)=n_{0}\left(E_{\mathbf{F}}\right)+x \frac{\mathrm{d} Z\left(E_{\mathbf{F}}\right)}{\mathrm{d} E} .
$$

La chaleur spécifique électronique doit croître linéairement avec la concentration. Cet effet doit être important car $\mathrm{d} Z / \mathrm{d} E$ est grand. On peut alors déduire des mesures de chaleur spécifique, la valeur de $\mathrm{d} Z / \mathrm{d} E$ au niveau de Fermi et si l'on suppose que les déphasages $\eta_{l}$ pour $l \neq 2$ varient peu, la forme de l'état lié virtuel $\boldsymbol{d}$ au voisinage du niveau de Fermi.

Actuellement, une seule mesure est connue sur $\mathrm{Cu} \mathrm{Ni}$ [28]. Elle donne pour $\mathrm{d} Z / \mathrm{d} E$ la valeur $0,7 \mathrm{eV}^{-1}$ alors que la forme simple parabolique étudiée au $\$ 1.2 c$ donne $0,8 \mathrm{eV}^{-1}$ en prenant $W=2,5 \mathrm{eV}$ et $Z_{2}\left(E_{\mathrm{F}}\right)=9$. haute température suit une loi de Curie-Weiss (fig. 7) $\chi=\mathrm{C} /(T-\theta)$ avec $C$ correspondant à un spin atomique $S=2, \theta$ étant proportionnel à la concentration $x$. A basse température, on voit apparaître un maximum de susceptibilité, pour une température voisine de $\theta$, un peu analogue à une transition graduelle vers l'antiferromagnétisme. De plus, on observe un petit moment ferromagnétique quand $T<\theta$.

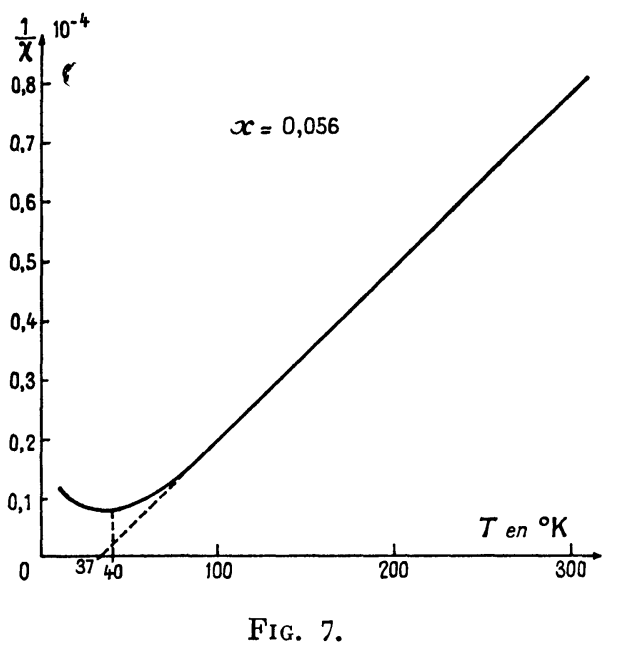

A basse température, $(T \ll \theta)$ on observe un déplacement de fréquences de résonance électronique très semblable aux effets observés dans la résonance antiferromagnétique. De plus les mesures de résonance nucléaire sur le cuivre [2] dans un alliage très dilué $(0,03 \%$ de $\mathrm{Mn})$ donnent un élargissement très important de la raie d'absorption, sans déplacement observable de position.

L'ensemble de ces résultats indique l'existence de couplages à longue distance entre atomes de manganèse, susceptibles de provoquer à basse température un "ordre " magnétique des atomes de manganèse.

2.2. Modìle D'interaction. - a) Interaction entre deux impuretés. - Soit un état lié virtuel avec découplage correspondant à une charge d'écran $2 Z$. Si l'on suppose que le potentiel dépendant du spin est négligeable à l'extérieur de la sphère atomique, on peut calculer la variation de charge déplacée à l'extérieur de la sphère, par l'atome d'impureté pour chaque direction de spin.

La partie radiale de la fonction d'onde d'un électron d'énergie $E=k^{2} / 2$ est :

$$
R_{l}(r)=A_{l}\left[\cos \eta_{l}^{\uparrow} j_{l}(k r)-\sin \eta_{l}^{\uparrow} n_{l}(k r)\right]
$$

pour une direction de spin ( $j_{l}$ et $n_{l}$ sont les fonctions de Bessel sphériques). 
La densité de charge déplacée est alors :

$$
\begin{array}{r}
\Delta \rho \uparrow(r)=\frac{1}{2 \pi^{2}} \sum_{\rho}(2 l+1) \int_{0}^{k_{F}} k^{2}\left[\sin ^{2} \eta_{l} \uparrow\left(n_{l}^{2}-j_{l}^{2}\right)\right. \\
\left.-\sin 2 \eta_{l}^{\uparrow} j_{l} n_{l}\right] \mathrm{d} k .
\end{array}
$$

Pour un état lié virtuel $d$ totalement découplé, $\eta_{l}{ }^{\downarrow}=0$ pour tout $l, \eta_{l}{ }^{\uparrow}=0$ pour $l \neq 2$. Alors $\Delta \rho^{\downarrow}=0$ et $\Delta \rho^{\uparrow}$ représente la différence des densités des électrons de spins opposés $\Delta \rho$ :

$\Delta \rho(r)=\frac{5}{2 \pi^{2}} \int_{0}^{k_{\mathrm{F}}} k^{2}\left[\sin ^{2} \eta_{2}^{\uparrow}\left(n_{2}^{2}-J_{2}^{2}\right)-\sin 2 \eta_{2}^{\uparrow} j_{2} n_{2}\right] \mathrm{d} k$.

Pour $r$ grand, on retrouve la formule donnée précédemment [20] :

$$
\Delta \rho(r)=\frac{5}{2 \pi^{2} r^{2}} \int_{0}^{k_{F}} \sin \eta_{2}^{\uparrow}(k) \sin \left[2 k R+\eta_{2}^{\uparrow}(k)\right] \mathrm{d} k .
$$

En prenant $2 Z=5$, et en supposant que $\eta_{2}^{\uparrow}(k)$ est linéaire en fonction de $\mathrm{k}$ sur un intervalle de largeur $k_{1},(f i g .8)$ on trouve pour $\Delta \rho(r)$ :

$$
\Delta \rho(r)=\frac{5}{4 \pi r^{3}} \frac{\sin k_{1} r \cos 2 k_{0} r}{k_{1} r+\pi}
$$

$k_{0}$ étant le vecteur d'onde moyen de l'état lié virtuel, $k_{1}$ sa largeur en $k(f i g .8)\left({ }^{6}\right)$. C'est une

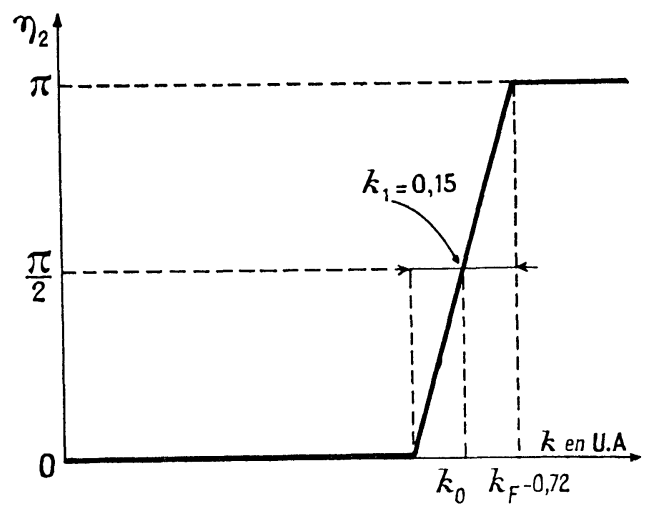

FIG. 8.

fonction oscillante, décroissant assez rapidement avec une période de l'ordre des dimensions interatomiques, ne dépendant pratiquement que de la largeur de l'état lié virtuel. Dans le cas réel; on aurait une fonction plus compliquée, mais de comportement analogue.

Si deux atomes 1 et 2 sont placés à la distance $R$, ils peuvent diffuser parallèlement ou antiparallèlement les électrons de la bande de conductibilité. Si $\Delta F$ est l'énergie d'échange entre un électron de la couche incomplète de spin total $\mathbf{S}$ et un électron de spin s, dans l'atome libre, l'énergie

(6) Dans la suite de l'exposé, les applications numériques sont faites en prenant $2 Z=5$ et la forme d'état lié virtuel d donnée par la figure 8 avec $k_{1}=0,15$ correspondant à un état lié virtuel de largeur $2 \mathrm{eV}$, d'échange $s-d$ dans le métal sera du même ordre. La différence d'énergie entre les configurations où les atomes ont leurs spins parallèles ou antiparallèles est alors :

$$
\Delta \varepsilon=2 \Delta \rho(r) v_{\mathbf{A}} \Delta F
$$

où $v_{\mathrm{A}}$ est le volume atomique.

En assimilant les états liés virtuels des atomes 1 et 2 à des couches atomiques de spin $\mathbf{S}_{1}$ et $\mathbf{S}_{2}\left({ }^{7}\right)$, l'interaction entre les atomes peut être mise sous la forme classique :

$$
J_{12} \mathbf{S}_{1} \cdot \mathbf{S}_{2} \text { avec } J_{12}=\frac{\Delta \rho(r) v_{\mathrm{A}} \Delta F}{2 S\left(S+\frac{1}{2}\right)} .
$$

b) Discussion du modèle d'interaction. - La question est de savoir quel est l'ordre de grandeur de l'interaction proposée, en particulier par rapport à l'interaction indirecte entre spins atomiques, par l'intermédiaire des électrons de conduction (du type Zener) [21].

Cette dernière interaction donne lieu à un couplage du type $J^{\prime}{ }_{12} \mathbf{S}_{1} \cdot \mathbf{S}_{2}$ avec

$$
J_{12}^{\prime}=\frac{9 \pi}{8} \frac{A^{2}}{E_{\mathrm{F}}} \frac{2 k_{\mathrm{F}} r \cos 2 k_{\mathrm{F}} r-\sin 2 k_{\mathrm{F}} r}{\left(2 k_{\mathrm{F}} r\right)^{4}}
$$

$E_{\mathrm{F}}$ est le niveau de Fermi.

$A$ la constante de couplage de l'interaction $s-d$

$$
A=\frac{\Delta F}{S+\frac{1}{2}} \text {. }
$$

$r$ la distance entre les atomes 1 et 2 .

On voit facilement que pour les distances qui nous intéressent (de 0 à $30 \AA$ distance moyenne entre proche voisins magnétiques pour une concentration de l'ordre de $0,1 \%$ ), le couplage $J_{12}$ par état lié virtuel est supérieur par un facteur de l'ordre de 5 à 15 à l'interaction $J^{\prime}{ }_{12}$ par couplage $s-d$. Il a de plus l'avantage de traiter les électrons de conduction sans supposer faible leur interaction avec l'impureté magnétique.

D'autre part, l'interaction d'échange entre électrons $s$ a été négligée. Les variations de densités d'électrons des deux directions de spins s'étendent sur tout le cristal. Mais on sait [22] que l'intervention de l'énergie d'échange a pour effet de stabiliser les différences de densités. Elle renforce donc un peu le couplage $J_{12}$.

Remarque. - Le déplacement de Knight dans les alliages $\mathrm{Cu} \mathrm{Mn}$.

L'élargissement de la raie de résonance nucléaire du cuivre a été expliqué par un couplage du type $K_{12} \mathbf{I}_{1} \mathbf{S}_{2}$ [23], entre le spin nucléaire $\mathbf{I}_{1} \mathrm{du}$ noyau $\mathrm{Cu}$ et le spin atomique $\mathbf{S}_{2}$ d'un atome $\mathbf{M n}$, $K_{12}$ est proportionnel à la variation de densité électronique des deux directions de spins

(7) En fait un état lié virtuel ne correspond pas à un spin bien défini, mais les résultats qualitatifs seront peu modifiés par cette hypothèse. 
$(\rho \uparrow-\rho \downarrow)$ donc à $A$ dans le cas du couplage Zener $s$ - $d$. Mais les élargissements observés correspondent à une valeur de $A$ dans $K_{12}$ au moins deux fois la valeur mesurée pour l'atome libre. Les variations de $(\rho \uparrow-\rho \downarrow)$ dues au niveau lié virtuel discutées plus haut doivent pouvoir mieux expliquer cet élargissement, mais aucun calcul numérique n'a été fait jusqu'ici.

\subsection{Applichtion a L'antiferromagnétique} DES ALLIAGES MÉTAL NOBLE-MÉTAL DE TRANSITION. - Seuls sont considérés ici les alliages parfaitement désordonnés (atomes répartis au hasard), de concentration $x$ en atomes magnétiques. Deux atomes magnétiques $i$ et $\mathrm{j}$ interagissent par un couplage du type Heisenberg $J_{\imath} \mathbf{S}_{\imath} . \mathbf{S}_{\jmath}, J_{\imath}$ étant une fonction oscillante de la distance séparant les atomes $i$ et $j$. L'état de plus basse énergie pour ces deux atomes peut être, soit parallèle, soit antiparallèle, suivant le signe de $J_{\imath \vartheta}$.

Pour un alliage de faible concentration, la distance moyenne entre atomes magnétiques est grande et $J_{\imath}$ a autant de probabilité d'être positif que négatif. Si on considère l'ensemble des atomes magnétiques, il y a autant d'atomes ayant leurs spins parallèles ou antiparallèles à une direction fixe de l'espace, la distribution des directions de spins étant fixée de telle sorte que l'énergie soit minima. On a ainsi une sorte d'antiferromagnétisme, où l'on distingue à l'état fondamental deux types d'atomes, sans avoir de surstructure magnétique : le désordre des spins est "gelé " à basse température.

L'état statistique de ce modèle est difficile car il faut traiter un phénomène d'ordre et désordre magnétiques sur un ensemble d'atomes qui ne sont pas équivalents. Deux méthodes sont utilisées : l'une s'applique aux hautes températures et permet de connaître la température de Curie paramagnétique; l'autre étudie le phénomène d'antiferromagnétisme à basse température.

a) Hautes températures. - Avec un couplage du type $J_{\imath j} \mathbf{S}_{\imath} \mathbf{S}_{\jmath}$, on obtient un développement en $1 / T$ de la susceptibilité par atome par la méthode d'Opechovski [24]

$$
\frac{1}{\chi}=\frac{3 K}{4 \mu^{2} S(S+1)}\left[T-y \frac{J}{2 K}+y(1+y) \frac{L^{2}}{4 K^{2} T}+\mathrm{O}\left(\frac{1}{T^{2}}\right)\right]
$$

où $\mu$ est le magnéton de Bohr, $T$ la température absolue, $K$ la constante de Boltzmann, $S$ le spin de l'atome magnétique,

$$
\begin{gathered}
y=\frac{4}{3} S(S+1) x \\
\left\{\begin{array}{c}
J=\sum_{\mathbf{B}} J_{0 \mathrm{~B}} \\
L^{2}=\sum_{\mathbf{B}} J_{0 \mathrm{~B}}^{2}
\end{array}\right.
\end{gathered}
$$

les sommes sont étendues à tous les sites $\mathrm{B}$ du cristal. $J_{\text {OB }}$ est l'intégrale d'échange entre deux atomes magnétiques situés aux sites $\mathrm{O}$ et $\mathrm{B}$ du cristal.

$$
J_{\mathrm{OB}}=\frac{A}{2 S} \int \Delta \rho(r) \mathrm{d} v \text { (l'intégrale est prise sur le }
$$
volume atomique de l'atome $B$; on suppose ici que la densité d'électrons $d$ est uniforme dans ce volume).

J peut donc être calculé : $J=\frac{A}{2 S} \int \Delta \rho(r) \mathrm{d} v$ (l'intégrale étant étendue à tout le volume extérieur à l'atome central $\mathrm{O}$ ).

La température de Curie paramagnétique $\theta=y \frac{J}{2 K}$ a alors la forme :

$\theta=\frac{5}{6} \frac{S(S+1)}{K} A r_{\boldsymbol{s}}^{2} x$

$\int_{0}^{k_{\mathrm{F}}} k \mathrm{~d} k\left[\sin ^{2} \eta_{2}^{\uparrow}\left(J_{5 / 2}^{2}-J_{-5 / 2}^{2}-J_{3 / 2}^{3} J_{7 / 2}+J_{-3 / 2} J_{-7 / 2}\right)\right.$

$\left.-\sin 2 \eta_{2}^{\uparrow}\left(J_{5 / 2} J_{5 / 2}+\frac{1}{2} J_{3 / 2} J_{-7 / 2}+\frac{1}{2} J_{-3 / 2} J_{7 / 2}\right)\right]$

$\left(r_{s}\right.$ est le rayon de la sphère atomique. Les fonctions $J_{n}$ sont les fonctions de Bessel ordinaires prises avec l'argument $\left.k r_{s}\right)$. Le terme principal, dans l'intégrale est le terme en $\sin ^{2} \eta_{2} \uparrow$ qui a un coefficient positif et variant lentement. Le second terme est le produit de $\sin 2 \eta_{2} \uparrow$ qui est une fonction à moyenne nulle par une fonction négative à faible variation. En prenant la forme d'état lié virtuel représenté sur la figure 8 pour $S=5 / 2$, on peut conclure un ordre de grandeur de $\theta$ :

$$
\theta \simeq 1000 x \text { en }{ }^{\circ} \mathrm{K}
$$

$\theta$ est donc positif, proportionnel à la concentration : expérimentalement, ceci est observé avec une constante de l'ordre de 900 [2].

Dans le développement de $1 / \chi$ le troisième terme est positif proportionnel à $x$ : il diminue toujours la susceptibilité ; les écarts à la loi de susceptibilité de haute température $\chi=\frac{\text { constante }}{T-\theta}$ sont effectivement observés dans ce sens. Ce terme en $1 / T$ est d'autant plus important pour une température relative $T / \theta$ donnée que la concentration est plus faible. Le développement de $1 / \chi$ en série de $1 / T$ est donc acceptable dans le domaine des hautes températures et des fortes concentrations.

b) Basses températures. - Il faut distinguer les alliages de fortes et de faibles concentrations. En effet, dans $J$, les termes les plus importants viennent des premiers voisins : en assimilant les douze premiers voisins de l'atome central à une couronne sphérique de même volume, on calcule aisément leur contribution qui est positive et représente la majeure partie de $J$. A basse température $\left(T<100{ }^{\circ} \mathrm{K}\right)$ on peut donc considérer que tous 
les couples de premiers voisins ont leurs spins parallèles.

On peut représenter l'alliage par des groupes de 1 , 2, 3 atomes interagissant par un couplage d'échange positif ou négatif. Il a été démontré [25] qu'il existe une concentration critique $x_{c}$ au-dessous de laquelle les impuretés ne forment que des "amas " finis, et au-dessus de laquelle il existe des "amas" infinis (on appelle amas tout groupe d'atomes d'impuretés proches voisins). Pour un réseau cubique à faces centrées (comme le réseau de cuivre, ou de l'or) $x_{c}=0,162$. Pour une concentratiòn supérieure à $x_{c}$, l'interaction entre proches voisins étant positive et beaucoup plus grande que les autres interactions, on doit s'attendre à ce que les amas infinis donnent lieu au ferromagnétisme. C'est ce qui a été observé pour les alliages $\mathbf{A u ~} \mathbf{M n}$ désordonnés aux concentrations $x=0,2$ et $x=0,25$ en atomes de manganèse $[26,[27]$.

$A$ faible concentration, $x \ll x_{\mathrm{c}}$ et basse température $\left(T<100^{\circ} \mathrm{K}\right)$, on peut d'autre part schématiser l'alliage de la façon suivante :

$N x(1-x z)$ moments permanents $\mu_{1}$ par unité de volume correspondant à un spin total.

$N x^{2} z / 2$ moments permanents $\mu_{2}$ par unité de volume correspondant à un spin $2 S$.

$N$ est le nombre d'atomes (magnétiques ou non) par unité de volume, $2 z$ le nombre de voisins dans le réseau cristallin,

$$
\mu_{1}=2 S \mu \quad \mu_{2}=4 S \mu .
$$

On ne tiendra pas compte des groupes de 3,4 atomes voisins entre eux,' car on va montrer que même les groupes de 2 n'ont que peu d'importance si $x$ est suffisamment petit.

Chacun des moments est soumis au champ magnétique appliqué $H$ et à un champ moléculaire $H_{\mathbf{M}}=+\left(M^{+}-M^{-}\right)$où $M^{+}$et $M^{-}$sont les aimantations des moments parallèles et antiparallèles au champ par unité de volume et $\mathrm{n}$ un coefficient de champ moléculaire. Dans les alliages suffisamment dilués étudiés ici, on peut confondre l'alliage avec un milieu continu : alors $n$, qui est un coefficient moyen, est indépendant de la concentration. Si l'on appelle $M_{1}^{+}, M_{1}^{-}, M_{2}^{+}, M_{2}^{-}$les aimantations totales des moments $\mu_{1}$ et $\mu_{2}$ parallèles et antiparallèles au champ $H$ par unité de volume, on a :

$$
\begin{aligned}
& M^{+}=M_{1}^{+}+M_{2}^{+} \\
& M^{-}=M_{1}^{-}+M_{2}^{-} \\
& M_{1}^{+}=\frac{N x}{2}(1-x z) \mu_{1} B_{S}\left[\frac{\mu_{1}}{k T}\left(H+n M^{+}-n M^{-}\right)\right] \\
& M_{1}^{-}=\frac{N x}{2}(1-x z) \mu_{1} B_{S}\left[\frac{\mu_{1}}{k T}\left(H+n M^{-}-n M^{+}\right)\right] \\
& M_{2}^{+}=\frac{N x^{2} z}{4} \mu_{2} B_{2 S}\left[\frac{\mu_{2}}{k T}\left(H+n M^{+}-n M^{-}\right)\right]
\end{aligned}
$$

$$
\begin{aligned}
& M_{2}^{-}=\frac{N x^{2} z}{4} \mu_{2} B_{2 S}\left[\frac{\mu_{2}}{k T}\left(\dot{H}+n M^{-}-n M^{+}\right)\right] \\
& B_{S} \text { et } B_{2 S} \text { sont les fonctions de Brillouin. }
\end{aligned}
$$

Quand $H$ est nul, $M^{+}$et $M^{-}$sont nuls audessus d'une certaine température de Néel $T_{\mathbf{N}}$ définie par

$$
k T_{\mathbb{N}}=\frac{4}{3} N n x \mu^{2} S(S+1)\left[1+\frac{S}{S+1} x z\right] .
$$

Le second terme de la parenthèse qui provient des groupes de deux atomes voisins vaut 0,20 pour $x=0,05$ et peut être négligé. Ainsi, alors que le comportement à haute température est déterminé par l'interaction entre proches voisins et par les couples de voisins, ceux-ci ne jouent que peu de rôle à basse température.

La température $T_{N}$ est alors proportionnelle à la concentration. Si l'on définit un nombre de " voisins effectifs " $2 z^{\prime}$ qui est le nombre de voisins qui créeraient le même champ moléculaire moyen, on a :

$$
z^{\prime} j(x)=2 n N x \mu^{2}
$$

où $j(x)$ est la valeur moyenne de $|J(r)|$, pour la distance moyenne entre voisins magnétiques à la concentration $x$ (pour $x$ petit)

$$
\jmath(x)=\frac{5 A}{16 \pi k_{1} S} \frac{x}{a x^{-1 / 3}+\frac{\pi}{k_{1}}}
$$

$a$ est la distance interatomique dans le cuivre.

En prenant pour $T_{\mathbb{N}}$, les valeurs expérimentales des températures pour lesquelles la susceptibilitè est maxima, on trouve $2 z^{\prime} \simeq 7$ pour $x$ entre 0,001 et 0,1 ce qui est une valeur raisonnable.

En réalité, en supposant que l'approximation du champ moléculaire est valable, le coefficient de champ moléculaire varie d'un atome à l'autre. Cela peut expliquer pourquoi le maximum de susceptibilité est très plat, sans température de Néel caractérisée, comme il est usuel dans les antiferromagnétiques ordonné :

2.4. Conclusion. - Les interactions de résonance décrites dans la première partie conduisent à un couplage magnétique entre impuretés à grande distance nettement plus fort que les modèles proposés jusqu'ici. Dans le cas particulier de l'alliage cuivre-manganèse, on explique les fortes températures de Curie paramagnétiques $\theta$, positives et proportionnelles à la concentration et les phénomènes d'ordre magnétique à basse température qui disparaissent à une température de Néel proportionnelle à la concentration. Les valeurs trouvées pour $\theta$ et $T_{\mathrm{N}}$ sont en accord approximatif avec l'expérience. L'étude théorique du modèle proposé n'est pas complète ; les résultats obtenus jusqu'ici semblent justifier son emploi. 
Il serait intéressant de l'étendre à d'autres systèmes d'alliages pour lesquels les données expérimentales sont actuellement assez incomplètes.

\section{Appendice}

La variation de la largeur de l'état lié virtuel $d$ avec son énergie moyenne peut être évaluée sur un modèle simple de potentiel. Les calculs ont été faits en prenant un puits de potentiel sphérique de rayon égal au rayon rs de la sphère atomique de la matrice et de profondeur variable. Les courbes de la figure 9 donnent les variations des déphasages en

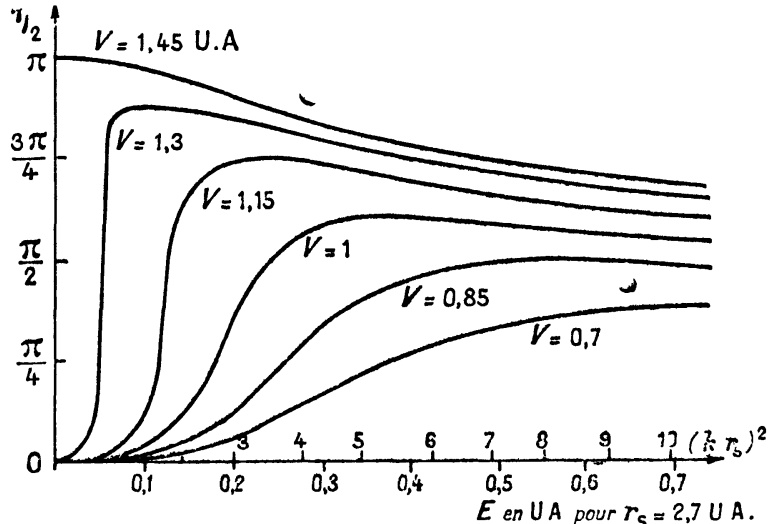

Fig. 9. - Déphasages $d$ produits par un puits de potentiel.

fonction de $\left(k r_{s}\right)^{2}$. Tous les métaux de même valence ont dans cette échelle le même niveau de Fermi. On définit l'énergie moyenne $E_{0}$ de l'état lié virtuel comme la valeur de l'énergie pour laquelle la courbe présente un point d'inflexion. La largeur $\mathbf{v}$ est prise égale à l'intervalle d'énergie $\left(E^{\prime \prime}-E^{\prime}\right)$ où $E^{\prime \prime}$ et $E^{\prime}$ sont donnés par

$$
\eta_{2}\left(E^{\prime}\right)=\frac{1}{2} \eta_{2}\left(E_{0}\right) \text { et } \eta_{2}\left(E^{\prime \prime}\right)=\frac{3}{2} \eta_{2}\left(E_{0}\right) \text {. }
$$

On voit alors que la largeur est proportionnelle à l'énergie moyenne $\boldsymbol{E}_{\mathbf{0}}$. Comme le montre le tableau suivant :

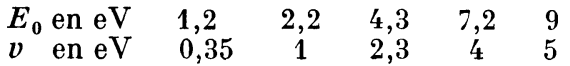

Il y a lieu de remarquer sur la figure 9 que les valeurs maxima de $\eta_{2}$ s'écartent nettement de la valeur $\pi$ quand l'énergie moyenne $E_{0}$ croît. Il n'en a pas été tenu compte dans l'exposé, mais cet effet doit diminuer la charge d'écran par les électrons $d$.

\section{RÉFÉRENCES}

[1] Owen (J.), Browne (M. E.), Knight (W. D.) et Kittel (G.), Phys. Rev., 1956, 102, 1501.

[2] Owen (J.), Browne (M. E.), Arp (V.) et Kip (A. F.) J. Phys. Chem. Solids, 1957, 2, 85.

[3] Schmitt (R. N.) et JAGons (I. S.), J. Phys. Chem, Solids, 1957, 3, 324.

[4] Gerritsen (A. N.) et Linde (J. O.), Physica, 1951, $17,573$.

[5] Gerritsen (A. N.) et Linde (J. O.), Physica, 1952, $18,877$.

[6] De Fajet de Casteluau (P.) et Friedel (J.), J. Physique Rad., 1956, 17, 27.

[7] Friedel (J.), Canad. J. Phys., 1956, 34, 1190.

[8] Huang (K.), Proc. Phys. Soc., 1948, 60, 161.

[9] Fö̈х (G.) et Wucher J. G.), C. R. A. S., 1954, 238, 1283.

[10] Fö̈x (G.) et Wucher (J. G.), J. Physique Rad., 1965, 17, 454 .

[11] Vogt (F.), Appl. Sc. Research., 1954, B 4, 1

[12] Friedel (J.), J. Physique Rad., 1958, 19, 573.

[13] Néel (L.), J. Physique Rad., 1932, 3,160.

[14] Kronevist (E.) et Giansoldati (A.), Archio Physik, $1953,7,343$.

[15] Vogt (E.), Z. Metal, 1951, 42, 155.

[16] Voft (E.) et Kruger, Ann. Physik, 1933, 18, 755.

[17] Kaufmann (A. R.) et Starr (C.), Phys. Rev., 1933, 63, 445 .

[18] Pugh (E.), Technical Report, 1956, 7 Carnegie Institute of Technology.

[19] Weil (L.), Thèse, Université de Strasbourg, Grenoble, 1941.

[20] Friedel (J.), Nuovo Cimento, 1958, 7, 287.

[21] Yosid a (K.), Phys. Rev., 1957, 106, 893.

22] Pines (D.), Solid State Physics, 1955, 1, 368.

[23] Behringer (R. F.), J. Phys. Chem. Sollds, 1957, $2,209$.

[24] Opechowski (W.), Physica, 1937, 4, 181.

[25] Lafore (P.) et De Gennes (P. G.), Rapport S. P. M. no 447 Centre ćnergie Atomique, J. Phys. Chem. Soldds (sous presse) '

[26] Meyer (A), C.R.A. S., 1956, 242, 2315.

[27] Meyer (A.), C.R.A.S., 1957, 244, 2038.

[28] Keesom (W. H.) et Kurrelmeyer, Physica, 1940, 7, 1003 . 